\title{
Understanding the Structure of Reversible Coordination Polymers Based on Europium in Electrostatic Assemblies Using Time Resolved Luminescence
}

Limin Xu, Mengqi Xie, Jianbin Huang* and Yun Yan*

Beijing National Laboratory for Molecular Sciences (BNLMS), State Key Laboratory for Structural Chemistry of Unstable and Stable Species, College of Chemistry and Molecular Engineering, Peking University, Beijing 100871, China

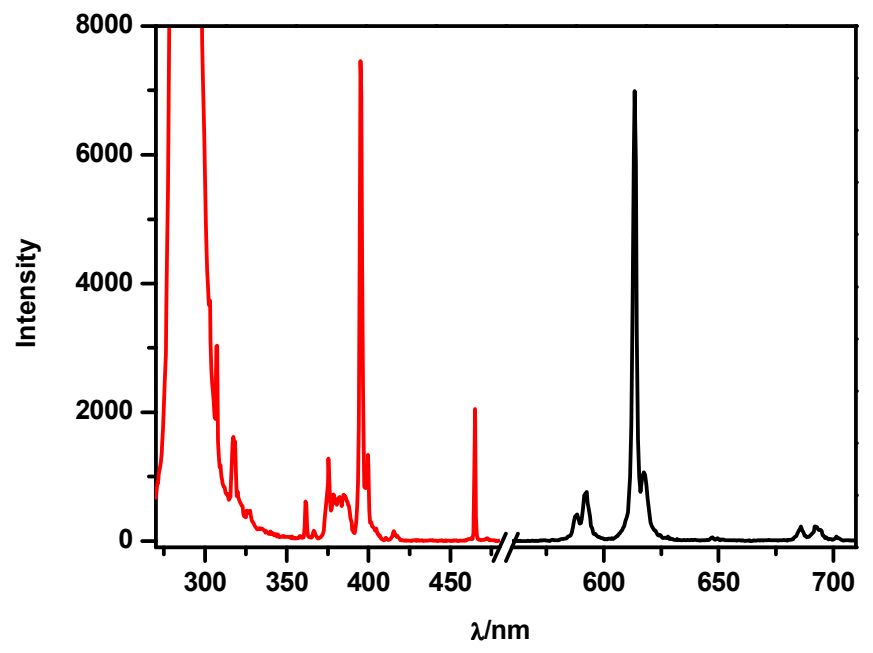

Figure S1.The excitation and emission spectra of $\mathrm{L}_{2} \mathrm{EO}_{4}-\mathrm{Eu}$ solution $\left(\left[\mathrm{Eu}^{3+}\right]=0.67 \mathrm{mM}, \mathrm{pH}=6.9\right.$, $\mathrm{L}_{2} \mathrm{EO}_{4} / \mathrm{Eu}=3 / 1$ ). The four peaks in excitation spectra at $317,362,395$ and $465 \mathrm{~nm}$ are attributed to ${ }^{7} \mathrm{~F}_{0}-{ }^{5} \mathrm{H}_{6} 、{ }^{7} \mathrm{~F}_{0}-{ }^{5} \mathrm{D}_{4} 、{ }^{7} \mathrm{~F}_{0}-{ }^{5} \mathrm{~L}_{6}$ and ${ }^{7} \mathrm{~F}_{0}-{ }^{5} \mathrm{D}_{2}$ transitions, respectively. The broad peak at $280 \mathrm{~nm}$ is attributed to ligand-metal charge transfer (LMCT). The three peaks in emission spectra around 595, 614 and $694 \mathrm{~nm}$ are attributed to ${ }^{5} \mathrm{D}_{0}-{ }^{7} \mathrm{~F}_{1} 、{ }^{5} \mathrm{D}_{0}-{ }^{7} \mathrm{~F}_{2}$ and ${ }^{5} \mathrm{D}_{0}{ }^{-7} \mathrm{~F}_{4}$ transitions, respectively. 

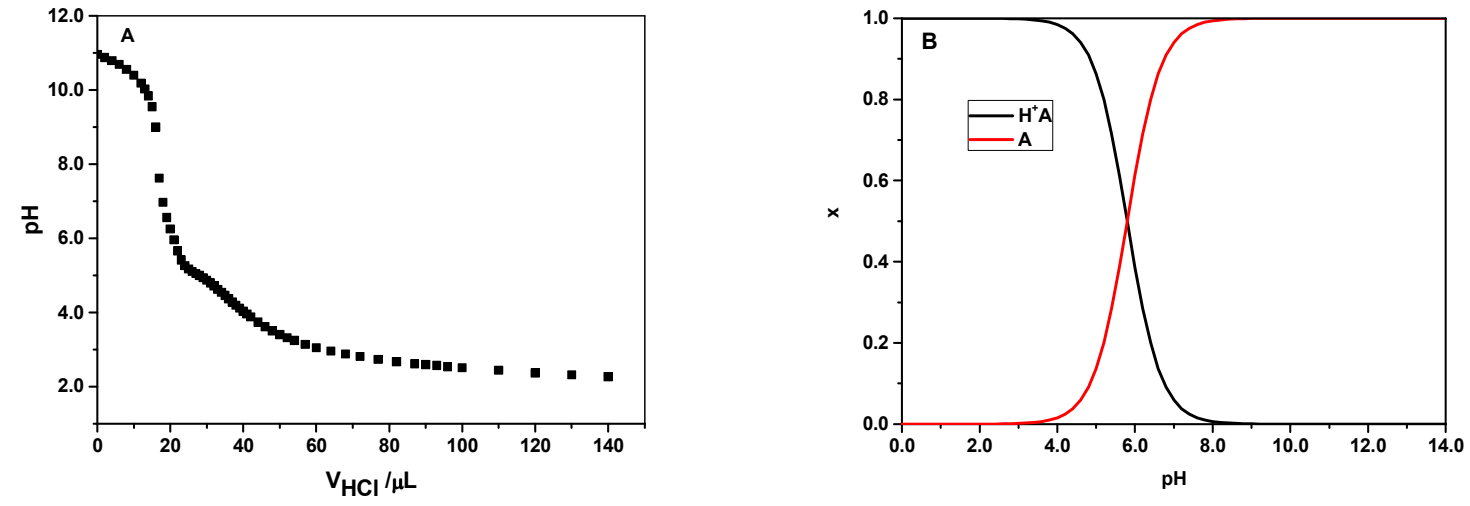

Figure S2.(A) Titration curve of $\mathrm{P}_{2} \mathrm{VP}_{41}-\mathrm{b}-\mathrm{PEO}_{205}$;(B) Curve of fraction of polymer versus $\mathrm{pH}$. $\left(\mathrm{A}=\mathrm{P}_{2} \mathrm{VP}_{41}-\mathrm{b}-\mathrm{PEO}_{205}, \mathrm{pKb}=8.2\right)$

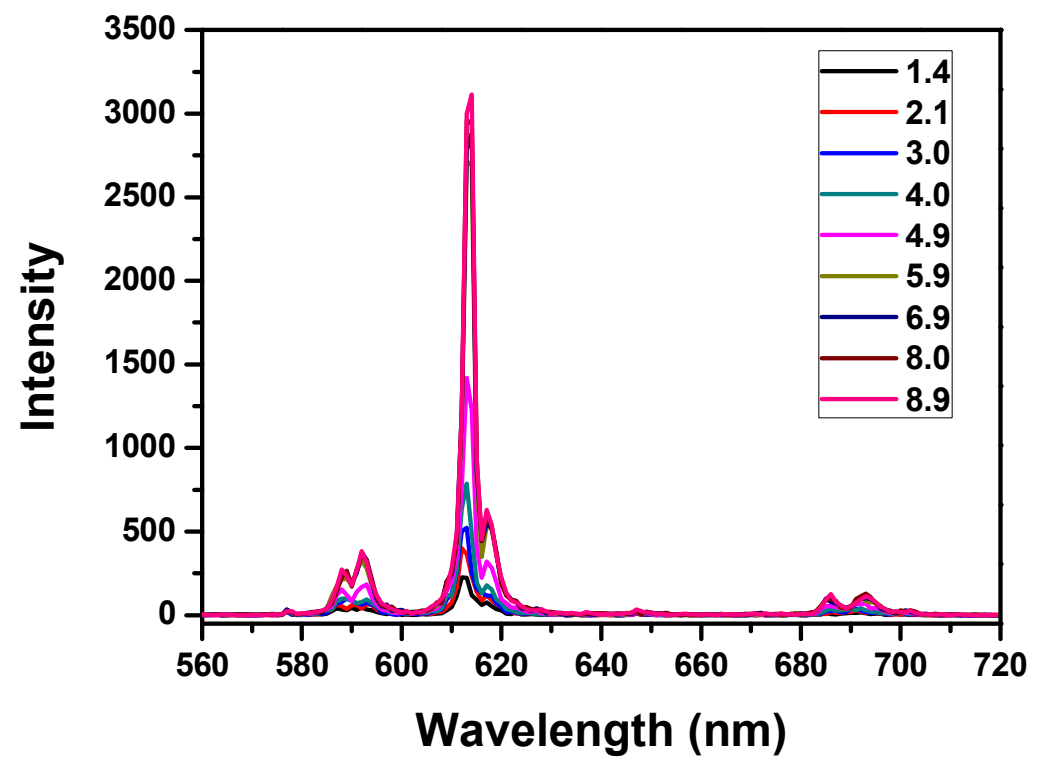

Figure S3. Emission spectra of $\mathrm{L}_{2} \mathrm{EO}_{4}-\mathrm{Eu}(3 / 2)$ complexes at different pH. $\left(\lambda_{\mathrm{ex}}=395 \mathrm{~nm},\left[\mathrm{Eu}^{3+}\right]=0.67 \mathrm{mM}, \mathrm{pH}=4.0\right)$. 


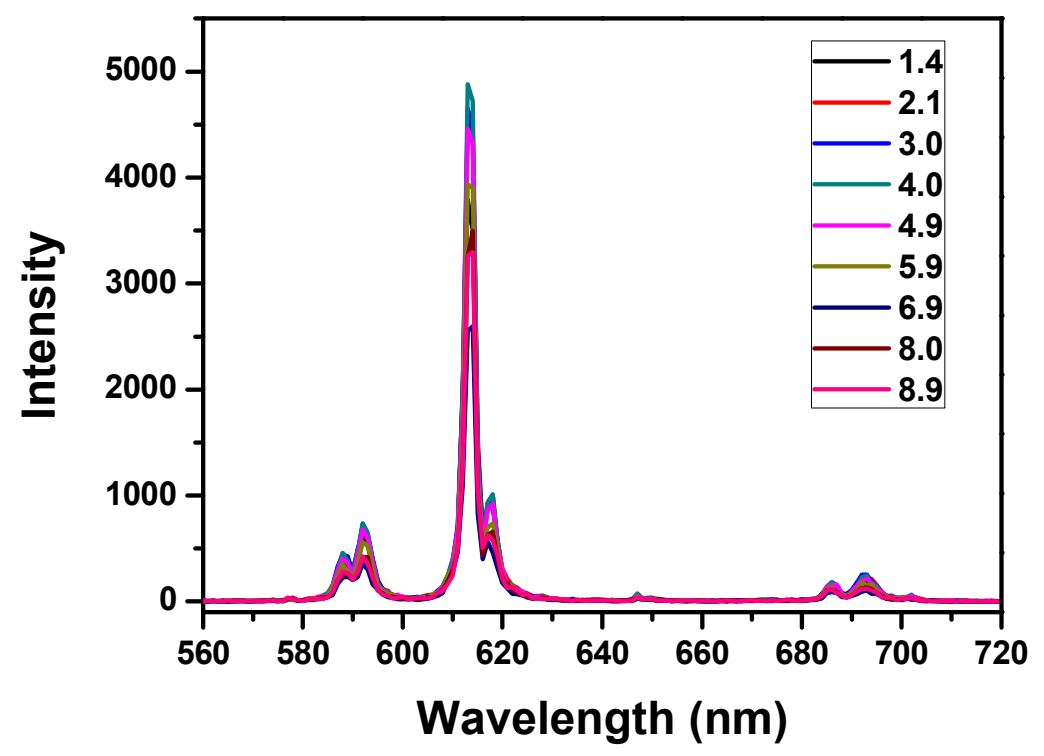

Figure S4. Emission spectra of $\mathrm{L}_{2} \mathrm{EO}_{4}-\mathrm{Eu}(3 / 2)$ complexes with $\mathrm{PVP}_{41}-\mathrm{b}-\mathrm{PEO}_{205}$ at different $\mathrm{pH}$. $\left(\lambda_{\mathrm{ex}}=395 \mathrm{~nm},\left[\mathrm{Eu}^{3+}\right]=0.67 \mathrm{mM}\right)$.

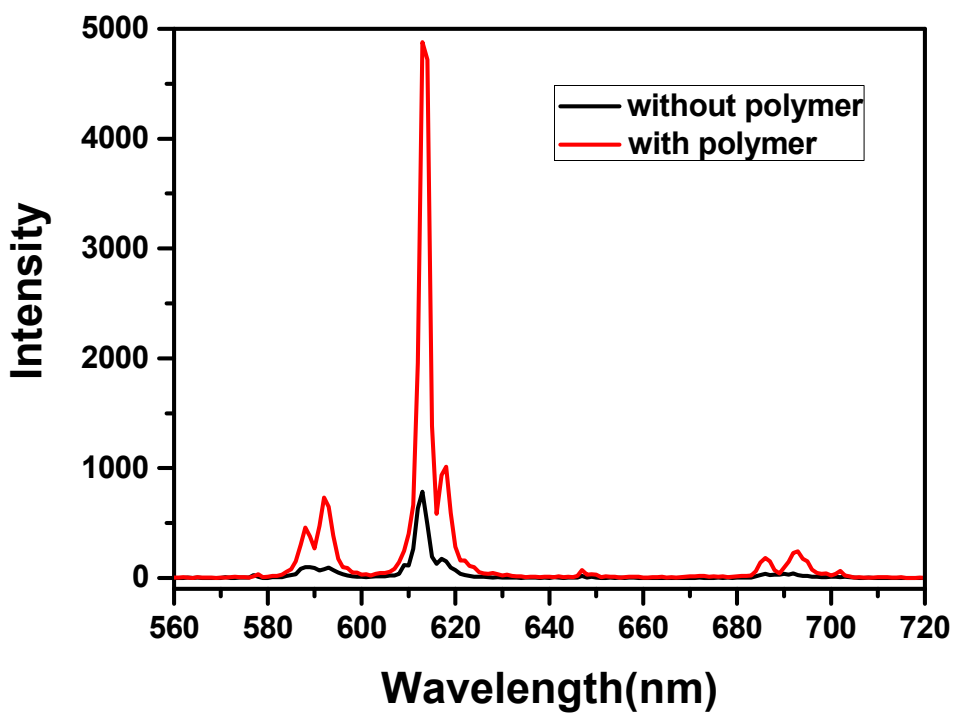


Figure S5.Emission spectra of $\mathrm{L}_{2} \mathrm{EO}_{4}-\mathrm{Eu}(3 / 2)$ complexes with and without $\mathrm{PVP}_{41}-\mathrm{b}-\mathrm{PEO}_{205} \cdot\left(\lambda_{\mathrm{ex}}\right.$ $\left.=395 \mathrm{~nm},\left[\mathrm{Eu}^{3+}\right]=0.67 \mathrm{mM}, \mathrm{pH}=4.0\right)$.

Table S1.Summary of evolutions of the transitions of $\mathrm{L}_{2} \mathrm{EO}_{4}-\mathrm{Eu}$ complexes in $\mathrm{H}_{2} \mathrm{O}$ $\left(\lambda_{\mathrm{ex}}=395 \mathrm{~nm},\left[\mathrm{Eu}^{3+}\right]=0.67 \mathrm{mM}, \mathrm{pH}=6.9\right)$

\begin{tabular}{|l|l|l|l|}
\hline & ${ }^{5} \mathrm{D}_{0}{ }^{-} \mathrm{F}_{1} / \mathrm{nm}$ & ${ }^{5} \mathrm{D}_{0}{ }^{-}{ }^{7} \mathrm{~F}_{2} / \mathrm{nm}$ & ${ }^{5} \mathrm{D}_{0}{ }^{-} \mathrm{F}_{4} / \mathrm{nm}$ \\
\hline $0 / 1$ & 593.0 & 613.0 & 693.5 \\
\hline $3 / 2$ & 593.5 & 612.5 & 692.0 \\
\hline $2 / 1$ & 592.0 & 613.5 & 693.0 \\
\hline $5 / 2$ & 592.0 & 613.5 & 693.0 \\
\hline $3 / 1$ & 592.5 & 613.5 & 693.0 \\
\hline $4 / 1$ & 592.0 & 613.5 & 692.5 \\
\hline $5 / 1$ & 592.0 & 613.5 & 692.5 \\
\hline
\end{tabular}

Table S2. Summary of evolutions of the transitions of $\mathrm{L}_{2} \mathrm{EO}_{4}-\mathrm{Eu}$ complexes with and without polymer in $\mathrm{H}_{2} \mathrm{O}$ at different $\mathrm{pH}\left(\lambda_{\mathrm{ex}}=395 \mathrm{~nm},\left[\mathrm{Eu}^{3+}\right]=0.67 \mathrm{mM}\right)$

\begin{tabular}{|c|c|c|c|c|c|c|}
\hline & \multicolumn{2}{|c|}{${ }^{5} \mathrm{D}_{0}{ }^{-} \mathrm{F}_{1} / \mathrm{nm}$} & \multicolumn{2}{c|}{${ }^{5} \mathrm{D}_{0}{ }^{7} \mathrm{~F}_{2} / \mathrm{nm}$} & \multicolumn{2}{c|}{${ }^{5} \mathrm{D}_{0}{ }^{7} \mathrm{~F}_{4} / \mathrm{nm}$} \\
\hline & complex & micelle & complex & micelle & complex & micelle \\
\hline 1.4 & 592 & 592 & 612 & 613 & 692 & 692 \\
\hline 2.0 & 593 & 592 & 612 & 613 & 691 & 692 \\
\hline 3.0 & 593 & 592 & 613 & 613 & 691 & 693 \\
\hline 4.0 & 593 & 592 & 613 & 613 & 692 & 693 \\
\hline 4.9 & 593 & 592 & 613 & 613 & 693 & 693 \\
\hline
\end{tabular}

